\title{
A COMPARATIVE SURVEY ON WIRELESS INTERNET USAGE AND FUTURE EXPECTATIONS OF UNIVERSITY STUDENTS (THE CASES OF USF,
AKU AND IUS)
}

Assoc. Prof. Hüseyin Koçak, Afyon Kocatepe University.

Assoc. Prof. Süleyman Dündar, Afyon Kocatepe University.

Prof.Dr. Hüseyin Arslan, University of South Florida.

\section{Abstract}

This article addresses the question of college students' internet usage and their expectations for better wireless access. Wireless and mobile internet access recently became an integral part of our lives. Especially given the introduction of smart devices (phones, tablets etc.) into the wireless market and the vast number of applications running on these devices, it is possible to become addicted to these devices. Some of us have come to a point where we can leave everything behind us except our phones. To better understand how wireless access has influenced us we conducted a face-toface survey with three different groups of college students totalling 829. According to the survey, there are some problems while using the internet, such as internet speed and connection. University students do not want to give up mobile communications and they never want to be secondary users. They pay more attention to security and privacy in communication. They are also awaiting functional innovations in future.

Keywords: Wierless Internet Usage; Security and Privacy; Cultural Differences; Social Reality and Technological Innovations. ${ }^{1}$ 


\section{INTRODUCTION}

Technology is transforming everything especially human relationships and thus the society and urban way of life at large. A wireless internet infrastructure with the capacity for fast data transfer is used in all areas from education to e-state and e-commerce. However, the rapid progress of internet and wireless communication gadgets has also brought about some problems with regard toadaptation to this technology. While evaluating wireless internet and communication, it is necessary that the progress of some national policies and the cultural structure be integrated with technological changes.

Mechanical timekeeping, cars, and mobile phones are technologies that have become embedded in our lives. These technologies challenged existing systems and firmly established themselves as inevitable for the functioning of society. The clock, the car and the mobile phone have all become central tools in our daily lives. From the mid-1990's onward, mobile communication became much more commercially accessible, and mobile technological innovations were rapidly accepted. Today, for most of us, it is impossible to think about a life without mobile phones, which began to enter our daily lives nearly 20 years ago. Similarly, mobile computers and wireless networks are becoming more integrated into our lives every day. Thus, it is not hard to estimate that the improvements in this area will continue in coming years. The relations between wireless communication and society are multi-dimensional.

Recent technological developments, particularly those experienced in internet technologies, have made the internet a primary tool for communication and access of information. Although the internet now figures in almost all areas of our lives, it is primarily used for communication and interaction. The internet provides educators with an incredible and rapidly developing knowledge base which makes it an indispensable tool to shape instructional endeavors. The rapid development and widespread use of the internet have also revealed some problems in integrating this new technology into the lifestyles of societies.

One purpose of this paper is to reveal the cultural differences affecting this adaptation directly or indirectly. The second purpose of this study is to bring together the expectations of the companies producing these technologies and the people using these technologies in an effective framework. In this context, innovations in internet and wireless communication can be presented by choice and diversity in accordance with countries, cultures and societies. It is possible that a wider acceptance of these products can be improved by considering the socio-economic aspects of these societies. In this paper, technology and society are discussed conceptually. Some historical information about technological developments is given, 
and after this, some technical questions are posed to students of The University of South Florida (USF), Afyon Kocetepe University (AKU) and Internatioanal University of Sarajevo (IUS). Students' opinions and expectations about internet usage are considered comparatively. This paper has a unique focus, as it engages with the technical field of social research as well as the social realities of these problems.

\section{CONCEPTUAL FRAMEWORK}

In this section, we provide a review of the existing literature on social and technical aspects of wireless communication technology.

\section{Social Research}

Technology does not determine society, it embodies it. Similarly, technological innovation is not determined by the society but instead, society simply uses it. This dialectical interaction between society and technology is present in the works of Fernand Braudel. Technological determinism is probably a false problem, since technology is society, and society can not be understood or represented without its technological tools. We live in the midst of a revolution in communication technologies that affects the way in which people feel, think and behave. Manuel Castells (2009) explores the nature of power itself in the new communications environment. He encompasses business, media, neuroscience, technology and, above all, politics.

Are mobile phones expressions of identity, fashionable gadgets, tools for life - or all of the above? 'Mobile Communications and Society' looks at how the possibility of multimodal communications from anywhere at any time affects everyday life at home, work, and school, and raises broader concerns about politics and culture both global and local. According to Castells (1998), wireless communication networks have been diffusing around the world faster than any other communication technology to date, because communication is at the heart of human activity in all spheres of life. He also asks some questions such as: does mobile communication favor the development of new youth culture that makes peer-to-peer networks the backbone of an alternative way of life? How does the interplay between new communication technologies and people's lives vary according to cultures and institutions in different regions of the world, and among different social groups?

Information and communication technologies are, furthermore, particularly sensitive to the effects of social users. The network society is also manifested in the transformation of sociability. Studies in different societies have indicated that in most instances internet users are more social, have more friends and contacts, and 
are more socially and politically active than non-users. Moreover, the more they use the internet, the more they also engage in face-to-face interaction in all domains of their lives. Similarly, new forms of wireless communication, from mobile phone voice communication to SMS, WiFi and Wimax, substantially increase sociability, particularly for younger age groups. The network society is a hypersocial society, not a society of isolation. However, there is a crucial change in sociability, not as a consequence of the internet or new communication technologies, but a change that is fully supported by the logic embedded in the communication networks. This is the emergence of networked individualism, as social structure and historical evolution induce the emergence of individualism as the dominant culture of societies. The networked society is a society of the networked individual (Castells, 1998).

Turkle (2011) has criticized the technology especially new mobile technologies. She asks: how are computers and internet changing us as a people? We are shaped by our tools. And now, the computer, a machine on the border of becoming a mind is changing and shaping us. Turkle asks how the self is being served. She informs us that the online self is flattened out into the impersonal. Privacy is gone. Attention is fragmented and shaped as we are urged to multitask, and provided with electronic mechanisms enabling us to do so. She also asserts that continuous connectivitiy afforded us by our machines does not bring us to a place of communion with each other, but rather isolates and distances us. She argues that aspects of technology cause addiction and dependency.

\section{Technical Research}

\section{Security \& Privacy}

As mobile internet-enabled devices become more pervasive in the homes and working lives of consumers, the demand to provide services traditionally available on desktop computers grows as well. Security is the most commonly cited reason for consumers' hesitation to use online services such as banking and shopping. It is not surprising, then, that concerns become even greater as we move from the wired desktop to wireless portable devices.

Security remains of primary importance for consumers and businesses that are using wireless services. To take full advantage of the wireless internet, businesses must understand the fundamental principles of security as to enable the implementation of a full range of wireless security measures. Proper security implementations will enable businesses to support their security claims and demonstrate to consumers that wireless transactions can be performed in a se- 
cure manner with respect to user validation, confidentiality, privacy, fraud and theft. It also lessens the business exposure cardless also extra costs incurred for offering services where, for instance, there are cardless transactions, making the wireless channel a much more compelling one on which to extend its services.

\section{Cognitive Radio}

Cognitive Radio (CR) is a very popular, extensively investigated, concept in wireless communication research, aimed at improving efficient usage of spectral resources. Flexibility, adaptation to the environment, and smart usage of spectral resources should be considered some of the key terms in this concept (Mitola et. al., 1999, pp. 13-18). A famous definition of CR that emphasizes its software-defined intellegence is 'brain empowered wireless communication (Haykin, 2005).

Enabling secondary users to utilize the licenced frequencies when the primary frequency owners are idle, dynamic spectrum accessing is one of the most important elements of CR technologies (Zhao, et. al., 2007, pp. 79-89). In order to facilitate secondary users to operate in that manner, many techniques and algorithms are developed such as adaptive waveform designes (Zhang, et. al., 2006), spectrum sensing algorithms (Yucek and Arslan, 2009), and spectral resource management techniques (Neel, 2006) in literature.

In today's wireless communication, a fixed spectrum assignment policy is implemented for each technology by authorities. Development of different technologies, increase in users's demands and inefficient usage of a limited spectrum enable a new communication network paradigm to emerge and rapidly improve. Cognitive radio networks, which are also known as next generation networks, aim to communicate continuously and rapidly as a result of using the spectrum efficiently and dynamically.

The demand for wireless access in voice and multi-media applications has also been increasing. As a result of the convergence of computing, content, and entertainment with communication, radio equipment has become part of our daily lives. It has come to a point where we can not live without them. We cannot interact, chat, find our way, have fun or think without them. We can leave everything else behind, but, cannot go anywhere without them. The fun is actually just starting, wait until you see intelligence added to these radios.

Today's wireless services have come a long way since the roll-out of the conventional voice-centric cellular systems. The demand for wireless access in voice and high rate data multi-media applications has been increasing too. New gen- 
eration wireless communication systems are aimed at accommodating this demand through better resource management and improved transmission technologies. The interest in increasing spectrum access and improving spectrum efficiency combined with both the introduction of software defined radios and the realization that machine learning can be applied to radios has created new intriguing possibilities for wireless radio researchers (Arslan, 2007).

Cognitive radio technology is a way in which one radio or even a network of radios are able to learn a useful degree of adaptivity, that aids the user, the network, and/or the spectrum owner. There are powerful economic incentives to provide new capabilities, through existing telecommunications infrastructure, and cognitive radios will provide those capabilities. As new services are offered, a larger spectrum will be needed and cognitive radios will provide the means for radios to communicate with greater spectrum efficiency (Bruce, 2007).

Dynamic spectrum access of primary users (PUs) and secondary users (SUs) is one of the most popular concepts in cognitive radio (CR) and promises to handle the congestion problem in spectrum. Considering the fact that most of the licenced spectrum usually becomes available during the daytime, SU concept is introduced to utilize licenced spectrum bands when their holders, i.e., PUs are idle. However, secondary users must leave the spectrum as soon as primary users appear. Therefore, they should monitor the spectrum in a periodic fashion to carry out the transmission without interfering with PUs and should only operate when a hole is sensed in the observed spectrum. If the entire spectrum is occupied, no action can be taken by SU and hence, risk of disconnection always exist for SUs. According to our survey, potential wireless customers do not give a credit such a usage, even if it is offered as a much cheaper price.

\section{Signal Seperation \& Interference Cancellation}

In wireless communication literature, signal seperation and interference cancellation are emerging techniques allowing signals to interfere with each other. If each receiver can cancel the alien signal or seperate an intended signal from the received mixture by performing some signal processing algorithm, actual data can be extracted properly. By this method, the aforementioned techniques allow multiple users to operate at the same time and frequency, and improves capacity by providing more spectral resource to each user. However, corresponding signal processing algorithms may introduce a significant complexity to the receiver device and may result in a degradation of battery life. According to our survey, potential wireless customers do not desire that situation even if their internet speed is considerably enhanced. 
Interference is one of the most critical issues limiting high data rates in wireless communication (Shin, et. al., 2007 and Russell, 1995). Most generally, it is caused by either another wireless signal or self-interference. In literature, interference avoidance (Lopez-Perez, 2009) and operation while allowing interference (Tarhini, Chadi and Tijani, 2007) are two popular approaches in dealing with this issue. However, since they need to allocate a significant part of the spectral resources for a reliable communication, they may not be considered spectrally efficient methods. In order to avoid extra spectral resource consumption unlike the aforementioned approaches, interference cancellation is an alternative method. Instead of compensating the effect of interference by introducing redundant information to the signal e.g., coding techniques, or deploying precautions such as guard band and guard time, interference is canceled at the receiver (Jeffrey, 2005). In addition to interference cancellation, various techniques such as full duplexing (Jain, et. al., 2011) and independent component analysis (ICA) (Mike and Christopher, 2007) are also being studied for facilitating the reception of more than one signal transmitted using the same spectral resource. However, these techniques introduce extra complexity and consume extra energy at the receiver. Therefore, when they are considered for wireless mobile devices, the life of the battery is degraded dramatically.

\section{Multi-Input Multi-Output (MiMo)}

Multi-Input Multi-Output (MIMO) is a communication technique in which the multipath properties of the channel are utilized to support greater data throughput. In a MIMO system, the transmitter transmits multiple channels of data traffic through multiple antennas: The receiver learns the channel behavior between the transmitter's multiple antennas and the receiver's multiple antennas, and uses signal processing to compute what waveform was transmitted by each transmitting antenna and the corresponding data stream. In this way, the same frequency is reused in the same geographic region to deliver greater amounts of data traffic than could be expected from a single transmitting and receiving antenna (SISO) system. Some MIMO systems also have the ability to learn to suppress interference from unrelated transmitters, further enhancing network performance. These MIMO techniques are practical when sufficient space for mounting antennas on the radio or platform are available (Bruce, 2007). Apart from technological advancement, MIMO systems have other practical projections onto the end-user's perspective such as battery life and the form factor of the mobile device utilizing multiples of antennas.

(MIMO) is increasingly used in wireless communication systems (Biglieri, 2010). The reason for this attention is that MIMO provides two types gain. First, for reducing the possibility of signal decay that will be sent simultaneously from differ- 
ent antennas, in radio channels of communication that will arise from the signal weakening that occur randomly (or failure). In other words, it enables the continuity of the signal from a fading antenna even stronger signals from other antennas -relatively- creating diversity enables mobile communication more smoothly. The other major advantages of MIMO is that the different signals from both antennas sending and receiving data traffic is provided in parallel with the number of antennas and communication speed can be increased proportionately (Lozano and Jindal, 2010, 186-197). However, these gains in multiple antenna radio channels have a critical requirement: size is comparable to the wavelength, independent of the distance between antennas (Chiani, 2003). That is the diversity of MIMO multiplexing gain of the wireless devices needs to be increased in size.

\section{RESEARCH OBJECTIVES}

Scientific research is performed to understand "social reality". One way of understanding social reality is to pose questions to the participants. The social impact of technological developments and innovations is one of the most studied topics in recent years. It was necessary for comparative research into the relationship between technological innovation and social needs to be conducted. Although technological developments are shaped by social needs, it is true that mankind's curiosity and motivation to achieve have a significant impact on these developments. When we look at developments in the internet and wireless technology, we see that it is characterised by consistently rapid changes. Within a short time, developments in the communications field has reached a very important stage. Wireless and mobile internet access have become an integral part of our lives In order to evaluate wireless internet usage by university students and their future expectations for future wireless service, a number of questions were put to three groups of university students, to establish the views and needs of these three different communities and their respective cultural structures. This survey was administered to college students from three different countries simultaneously. The main objective of this research is determining the habits of college students' internet usage and to get information about and expectations for future technology and its impact on the students' socio-cultural processes.

\section{Research universe and sampling}

In general, topics such as technology, society, and internet technology cover the research universe. In particular, it includes habits of college students' internet usage and expectations. The students of USF, IUS and AKU are the sample of the study. This survey was conducted in February 2014. 


\section{USF (The University of South Florida)}

The University of South Florida is a research university located in Tampa Bay on Florida's west coast. It is one of the largest public universities in the nation, and among the top 50 universities, public or private, for federal research expenditures. The university is one of only four Florida public universities classified by the Carnegie Foundation for the Advancement of Teaching in the top tier of research universities, a distinction attained by only 2.3 percent of all universities. The University of South Florida is serving nearly 48,000 students (www.usf.edu).

\section{AKU (Afyon Kocatepe University)}

Afyon Kocatepe University was founded in 1992 in Afyonkarahisar, Turkey, and started educational activities during the 1992-1993 academic year. Now, the university has educational activities with 12 faculties and 4 schools at the Bachelor of Science degree level, 15 vocational colleges at the Associate Degree level, 3 institutes for graduate education. Afyon Kocatepe University has more than 30000 registered student (www.aku.edu.tr).

\section{IUS (International University of Sarajevo)}

The International University of Sarajevo is located in Sarajevo, the capital of Bosnia-Herzegovina. During undergraduate and postgraduate studies, students are developing in a quality intellectual atmosphere and are able to choose one of the 14 programs of studies within the three faculties, containing programs in different areas ranging from industrial engineering and computer sciences art and social sciences and to business administration and humanities. There are more than 2000 students studying in the first, second and third cycle of studies, coming from all continents and approximately from 40 countries of the world (www.ius.edu.ba).

\section{METHODS AND TECHNIQUES USED IN THIS RESEARCH}

This study encompasses a 'micro-field survey' as well as a literature review and analysis of survey techniques. The first section of the research is theoretical, while the second partispractical. After reviewing the literature on thesubject, data were obtained to try to establish a general framework in the light of the research. This framework offers a theoretical premise and in order to test the hypotheses, a survey was completed by students.

\section{RESEARCH HYPOTHESIS}

1. Participants are aware that the Wi-Fi modem in their homes actually has a small base station function.

2. Participants do not prefer a service provider which gives a service with a 


\section{H. Koçak, et. al}

higher risk of connection failure but lower prices.

3. Participants want to use the fastest device to access the internet, regardless of size.

4. The mobility during communication is always important for the participants.

5. Participants would accept having less battery life (e.g., half) if they would have doubled internet speed the same price.

6. By paying more money, participants expect more secure personal communication carried out via email, social networks etc.

\section{FINDINGS AND INTERPRETATIONS}

Representative examples of our universe, our research group; 131 females and 139 males in USF, 142 females and 156 males in AKU and 42 females and, 219 males in IUS consists of a total of 829 people. All of these students answered the questions by filling out questionnaires. These responses were analyzed by statistical methods. Frequency tables, which were generally involved in the study, give the percentage of the sample group.

\section{Gender}

\begin{tabular}{|l|l|l|l|}
\hline Name of the University & $\begin{array}{l}\text { Response } \\
\text { Categories }\end{array}$ & Frequency & Percent \\
\hline \multirow{4}{*}{ USF } & Male & 139 & 51,5 \\
\cline { 2 - 4 } & Female & 131 & 48,5 \\
\cline { 2 - 4 } & Total & 270 & 100,0 \\
\hline \multirow{4}{*}{ AKU } & Male & 156 & 52,3 \\
\cline { 2 - 4 } & Female & 142 & 47,7 \\
\cline { 2 - 4 } & Total & 298 & 100,0 \\
\hline \multirow{3}{*}{ IUS } & Male & 219 & 83,9 \\
\cline { 2 - 4 } & Female & 42 & 16,1 \\
\cline { 2 - 4 } & Total & 261 & 100,0 \\
\hline
\end{tabular}

When looking at the gender distribution of the participants, it is clear that there is an equal distribution between males and females. However, the rates of distribution are: $51.5 \%$ male, $48.5 \%$ female for USF. These distribution are $52.3 \%$ male, $47.7 \%$ for AKU. These are $83.9 \%$ male and $16.1 \%$ female for IUS. Quite different from physical definition in our mind, gender issue is based on physcological and sociologic discrepancy as a scientific definition. Necessarily asked in social science research. The 
gender question has a determinant role in people's social lives, routines and decisions.

2.Areyou aware that the Wi-Fimodem in yourhomeis actually a smallbase station?

\begin{tabular}{|l|l|l|l|}
\hline Name of the University & Response Categories & Frequency & Percent \\
\hline \multirow{4}{*}{ USF } & Yes & 140 & 51,9 \\
\cline { 2 - 4 } & No & 52 & 19,3 \\
\cline { 2 - 4 } & No idea & 67 & 24,8 \\
\cline { 2 - 4 } & I don't have a modem & 11 & 4,1 \\
\cline { 2 - 4 } & Total & 270 & 100,0 \\
\hline AKU & Yes & 211 & 70,8 \\
\cline { 2 - 4 } & No & 38 & 12,8 \\
\cline { 2 - 4 } & No idea & 20 & 6,7 \\
\cline { 2 - 4 } & I don't have a modem & 29 & 9,7 \\
\cline { 2 - 4 } & Total & 298 & 100,0 \\
\hline \multirow{5}{*}{ IUS } & Yes & 118 & 45,2 \\
\cline { 2 - 4 } & No & 52 & 19,9 \\
\cline { 2 - 4 } & No idea & 74 & 28,4 \\
\cline { 2 - 4 } & I don't have a modem & 17 & 6,5 \\
\cline { 2 - 4 } & Total & 261 & 100,0 \\
\hline
\end{tabular}

The aim of this question is to learn if the people who think that base stations are dangerous under normal conditions, are aware that the modem which they have in their houses has the same function. Certainly, modems at home may not affect people's health in as much as the base station outside. On the other hand, is there any inconvenience to put a Femcotell (a device which provides the mobile phone with the modem at home rather than the base station outside) into the houses in the near future? In terms of deploying such a technology this, it might be considered as an important question. $51.9 \%$ of the USF students indicate that Wifi modems at their houses function as a small base stations. However, this rate is $70.8 \%$ for AKU students, it is $45.2 \%$ for IUS students. It might be said the most of the participants are aware that the mobile phones use the same technology with the base stations and Wifi. This case strengthen the possibility that applications like Femtocell will not create much of a reaction. 
3. Would you prefer a service provider which gives a service with a higher risk of connection failure but lower prices?

\begin{tabular}{|l|l|l|l|}
\hline Name of the University & Response Categories & Frequency & Percent \\
\hline \multirow{4}{*}{ USF } & Yes & 23 & 8,5 \\
\cline { 2 - 4 } & No & 247 & 91,5 \\
\cline { 2 - 4 } & Total & 270 & 100,0 \\
\hline \multirow{4}{*}{ AKU } & Yes & 38 & 12,8 \\
\cline { 2 - 4 } & No & 260 & 87,2 \\
\cline { 2 - 4 } & Total & 298 & 100,0 \\
\hline \multirow{4}{*}{} & Yes & 119 & 45,6 \\
\cline { 2 - 4 } & No & 142 & 54,4 \\
\cline { 2 - 4 } & Total & 261 & 100,0 \\
\hline
\end{tabular}

This question is being asked to understand how the society meets the secondary user concept in cognitive radio applications. In this concept users are classified into two groups called primary users (PUs) and secondary users (SUs). Primary users can be described as the owners of the spectral resources. For example, a frequency band is assigned to a PU for a given time period and he can use it whenever he needs. In this way, available spectral resources are shared between PUs in a geographical area. However, PUs do not always need to use these resources. Considering this fact, cognitive radio introduces secondary usage concept and allows users having no allocated spectral resource to operate in frequency bands of PUs when they are idle. However, secondary users have to leave the frequency band of a PU immediately when the PU start to use it. Therefore, communication of an SU might me interrupted by a PU and connection failure risk becomes quite higher even if it costs less than owning a spectral resource. USF students mostly gave no answers (91.5\%). A minority responded 'Yes, I prefer' (8.5\%). AKU students answered this question as $87.2 \%$ "no", 12.8\% “yes". IUS students presented opinions as 54.4\% "no", 45.6\% “yes" for this question. Here, the most different result belongs to IUS students. Among the IUS students, the rate of being secondary users is fairly high. The reason of this result may be the high price of internet in that country. On the other hand, it is seen that people generally don't prefer low price services with the secondary base station. Or rather, even if it is cheap, an operator which has a high risk of cessation is not preferred. 
4. Noting that the size of a smartphone is proportional to its data rate, I prefer a large smartphone with high data rate to a small one with a lower internet speed. (Please ignore the benefit of wider screens of the large phones in this question.)

\begin{tabular}{|l|l|l|l|}
\hline Name of the University & Response Categories & Frequency & Percent \\
\hline \multirow{4}{*}{ USF } & Totally agree & 9 & 3,3 \\
\cline { 2 - 4 } & Agree & 42 & 15,6 \\
\cline { 2 - 4 } & Neutral & 86 & 31,9 \\
\cline { 2 - 4 } & Disagree & 100 & 37,0 \\
\cline { 2 - 4 } & Totally disagree & 33 & 12,2 \\
\cline { 2 - 4 } & Total & 270 & 100,0 \\
\hline \multirow{5}{*}{ AKU } & Totally agree & 52 & 17,4 \\
\cline { 2 - 4 } & Agree & 75 & 25,2 \\
\cline { 2 - 4 } & Neutral & 45 & 15,1 \\
\cline { 2 - 4 } & Disagree & 76 & 25,5 \\
\cline { 2 - 4 } & Totally disagree & 50 & 16,8 \\
\cline { 2 - 4 } & Total & 298 & 100,0 \\
\hline \multirow{5}{*}{ IUS } & Totally agree & 21 & 8,0 \\
\cline { 2 - 4 } & Agree & 40 & 15,3 \\
\cline { 2 - 4 } & Neutral & 78 & 29,9 \\
\cline { 2 - 4 } & Disagree & 85 & 32,6 \\
\cline { 2 - 4 } & Totally disagree & 37 & 10,2 \\
\cline { 2 - 4 } & Total & 261 & 100,0 \\
\hline
\end{tabular}

The point of this question is to understand what will be the reaction of people to the deployment of wireless capacity increasing techniques that require more space on the devices such as MIMO (more antenna usage on the device). Usage of such technologies leads to increase of device sizes, naturally. How much will the people be content with this? $49.2 \%$ of students in USF, $42.3 \%$ of students in AKU, and $46.8 \%$ of students in IUS presented opinions as 'I disagree and stronly disagree. On the other hand, in this question, how much could the people isolate the screen advantage is also another important factor to consider. 
5. I prefer watching an online video over cellular network with moderate quality rather than waiting until I arrive at home/office for watching the same video over WiFi with high quality.

\begin{tabular}{|l|l|l|l|}
\hline Name of the University & Response Categories & Frequency & Percent \\
\hline \multirow{5}{*}{ USF } & Totally agree & 32 & 11,9 \\
\cline { 2 - 4 } & Agree & 96 & 35,6 \\
\cline { 2 - 4 } & Neutral & 63 & 23,3 \\
\hline \multirow{5}{*}{ AKU } & Disagree & 64 & 23,7 \\
\cline { 2 - 4 } & Totally disagree & 15 & 5,6 \\
\cline { 2 - 4 } & Total & 270 & 100,0 \\
\hline & Totally agree & 76 & 25,5 \\
\cline { 2 - 4 } & Agree & 102 & 34,2 \\
\cline { 2 - 4 } & Neutral & 36 & 12,1 \\
\cline { 2 - 4 } & Disagree & 64 & 21,5 \\
\cline { 2 - 4 } & Totally disagree & 20 & 6,7 \\
\cline { 2 - 4 } & Total & 298 & 100,0 \\
\hline \multirow{5}{*}{ IUS } & Totally agree & 15 & 5,7 \\
\cline { 2 - 4 } & Agree & 34 & 13,0 \\
\cline { 2 - 4 } & Neutral & 70 & 26,8 \\
\cline { 2 - 4 } & Disagree & 92 & 35,2 \\
\cline { 2 - 4 } & Totally disagree & 50 & 19,2 \\
\cline { 2 - 4 } & Total & 261 & 100,0 \\
\hline
\end{tabular}

While students in USF gave $47.5 \%$ 'strongly agree and agree' answers, 59.7\% of students in AKU and $18.7 \%$ of students in IUS gave 'strongly agree and agree' answers. Here, the most salient finding is the thoughts of the IUS students. $54.4 \%$ of IUS students remark that they don't agree with this idea. Interestingly, in this question it is seen that the answers have a balanced distribution. However, overall tendency shows even if it has lower quality, people will not give up mobile access.

If the user or device is moving, this can disrupt the received signals, and the reflection angle and other parameters can vary rapidly. If transmitters and receivers do not follow this change in surrounding; the quality of communication declines (Torbjorn, 2002). The effect of this reduction of the data rate is relatively low (8-15 $\mathrm{kbps}$ ) in voice communications, and is not noticeable. But anything that requires high data rate, real-time (video-over-IP), or video applications such as video-on-demand mobility directly affects the quality of service. On the other hand, support 
for mobile communication wireless local area networks (Wireless LANs) provides services with higher data rates. So from the local network to the cellular cellular service providers discharge data (data off-loading) is working on (Lee et. al., 2010).

In another study, even without taking into account the need to defer $65 \%$ of the cellular network to the local network traffic can be obtained discharge. By anticipating users' movements before there are studies that aim to realize in this off-loading in an efficient manner (Baier). Consequently, we also tried to predict by this question providing mobile services for cellular networks of multimedia applications and wireless local area networks due to mobility in the distribution of the share of the falling quality of service. Interestingly, the participants have approached this issue with a similar range. Nevertheless, there is a trend that people do not give up the ease of access even in a lower quality. At the same there are some users who are delaying the needs by loading transfer from the cellular network to the local network and these can not be underestimated.

6. I would accept having less battery life (e.g., half) if I am going to have doubled internet speed with the same price.

\begin{tabular}{|l|l|l|l|}
\hline Name of the University & Response Categories & Frequency & Percent \\
\hline \multirow{4}{*}{ USF } & Totally agree & 39 & 14,4 \\
\cline { 2 - 4 } & Agree & 106 & 39,3 \\
\cline { 2 - 4 } & Neutral & 61 & 22,6 \\
\cline { 2 - 4 } & Disagree & 51 & 18,9 \\
\cline { 2 - 4 } & Totally disagree & 13 & 4,8 \\
\cline { 2 - 4 } & Total & 270 & 100,0 \\
\hline AKU & Totally agree & 24 & 8,1 \\
\cline { 2 - 4 } & Agree & 49 & 16,4 \\
\cline { 2 - 4 } & Neutral & 50 & 16,8 \\
\cline { 2 - 4 } & Disagree & 101 & 33,9 \\
\cline { 2 - 4 } & Totally disagree & 74 & 24,8 \\
\cline { 2 - 4 } & Total & 298 & 100,0 \\
\hline \multirow{5}{*}{ IUS } & Totally agree & 21 & 8,0 \\
\cline { 2 - 4 } & Agree & 57 & 21,8 \\
\cline { 2 - 4 } & Neutral & 72 & 27,6 \\
\cline { 2 - 4 } & Disagree & 70 & 26,8 \\
\cline { 2 - 4 } & Totally disagree & 41 & 15,7 \\
\cline { 2 - 4 } & Total & 261 & \\
\hline
\end{tabular}


This question is to understand how wireless capacity increasing techniques that demands more energy comsumption will be accepted by the users. In this context, signal seperation and interference cancellation techniques are considered in this study. They are emerging techniques allowing signals to interfere with each other. If a receiver can seperate the intended signal from the received signal mixture by performing some signal processing algorithms, actual data can be extracted properly. If receivers can carry out these processes, we can allow multiple users to operate at the same time and frequency. Thus capacity is improved by providing more spectral resource to each user. However, aforemention signal processing algorithms introduce a significant complexity to the receiver device and result in a considerable degradation battery life. By evaluating the answers given to "Question-6", we discuss the future of these techniques.

While the $53.7 \%$ of USF studentsgive 'stronglyagree and agree'answers, this rate among AKU students is $24.5 \%$ and $29.8 \%$ for IUS students. Here, different views belong to USF students. Students of IUS and AKU gave similar answers. Although people mostly complain about the link speed, they do not want to be charged more to connect to the internet faster. It is seen that battery life maintains its importance for many people. It is possible to make two inferences: Studies to prolong battery life will maintain importance, and the producers should focus on decresing waste of energy in mobile devices.

\section{By paying more money, I want more secure personal communication carried} out via email, social networks etc.

\begin{tabular}{|l|l|l|l|}
\hline Name of the University & Response Categories & Frequency & Percent \\
\hline USF & Totally agree & 4 & 1,5 \\
\cline { 2 - 4 } & Agree & 22 & 8,1 \\
\cline { 2 - 4 } & Neutral & 65 & 24,1 \\
\cline { 2 - 4 } & Disagree & 105 & 38,9 \\
\cline { 2 - 4 } & Totally disagree & 74 & 27,4 \\
\cline { 2 - 4 } & Total & 270 & 100,0 \\
\hline AKU & Totally agree & 52 & 17,4 \\
\cline { 2 - 4 } & Agree & 87 & 29,2 \\
\cline { 2 - 4 } & Neutral & 67 & 22,5 \\
\cline { 2 - 4 } & Disagree & 59 & 19,8 \\
\cline { 2 - 4 } & Totally disagree & 33 & 100,0 \\
\cline { 2 - 4 } & Total & 298 & 1,1 \\
\hline
\end{tabular}




\begin{tabular}{|l|l|l|l|}
\hline \multirow{4}{*}{ IUS } & Totally agree & 8 & 3,1 \\
\cline { 2 - 4 } & Agree & 22 & 8,4 \\
\cline { 2 - 4 } & Neutral & 78 & 29,9 \\
\hline \multirow{4}{*}{} & Disagree & 101 & 38,7 \\
\cline { 2 - 4 } & Totally disagree & 52 & 19,9 \\
\cline { 2 - 4 } & Total & 261 & 100,0 \\
\hline
\end{tabular}

Security is a crucial task in wireless communication due to the broadcast nature of wireless signals. In order to achieve data protection, encryption based security techniques have been implemented so far. Recently, security precautions in physical layer (PHY) have also become popular and are being intensively investigated in literature. Unlike encryption techniques, PHY security deals with the secrecy of signal rather than the data. By this way, it provides a complementary protection in wireless communication. However, all of these techniques may require extra-spectral resource consumption and will likely cause a raise in wireless service charges. This question discusses the future demand for a secure communication at expense of paying more money.

The rate of the USF students who want to pay more in return to communicate securely is nearly $9.6 \%$. A similar rate with $11.5 \%$ belongs to IUS students. The different view about this topic belongs to AKU students. $46.6 \%$ of AKU students want to communicate securely paying more. In recent years, security problems in Turkey may have caused this result. As these results indicate, Turkish people prefer to pay more to provide secure communication. They concluded that the price is not important as long as a secure communication is provided.

\section{CONCLUSION}

Scientists think; engineers make. Engineering is fundamentally an activity, as opposed to an intellectual discipline. The goal of science and philosophy is to know; the goal of engineering is to do something good or useful. But even in that bare-bones description of engineering, the words good and useful have philosophical implications (Stephan, et. al., 2012). Society and communication is one of the most important issues in recent years.

The innovations in the field of wireless communication especially have developed very quickly. Social structure and urban life are affected by this change. Communities can benefit from innovations developed in the field of wireless communication and the enrichment of the otherwise backward. What important is not only the use of technology to produce it. In this context, science 
and technology policies should be revised by the corresponding authorities.

This research is one of the first into the area of social provision of technical problems, and has generated interesting results. Technical problems and prospects have been investigated through the questions posed by our survey. The researchers working in this field have contributed in the creation of technical questions. College students are preferred because they represent certain segments of society; they are more likely to use technological devices and more sensitive to these areas.

In this respect, theanswers from the threegroups of people from different societies have revealed that wireless innovations will continue to be one of the determining factors in social behavior. Although accepting the smartphone as a leading role in the personal life does not seem to be a widespread adoption, new technologies in wireless systems will still be the directors of the main elements in the personal and social life. Awareness of the tecnological developments and the end-user requirements that can be challenging in the technological perspective are among the evidences of this direct role.

In terms of the technical aspect, this study also investigated the future of current research topics in wireless communication technology by identifying the expectations of users. Since nothing comes for free, proposed improvements in wireless technology are also obtained at the expense of various disadvantages, e.g., degradation in battery life, higher service costs, increased device sizes etc. Our survey questions were prepared and posed to the college/university students, in order to compare the advantages and the trade-offs in aforementioned research fields. Considering the results of our survey, which clearly vary between different countries, the wireless industry can re-evaluate these research fields and can identify which technologies will be accepted more by which society, in the future. 


\section{REFERENCES}

Arslan, Huseyin (Ed.) 2007. Cognitive Radio, Software Defined Radio, and Adaptive Wireless Systems. Springer: The Netherlands.

Baier, Patrick; Dürr, Frank; Rothermel, Kurt. TOMP: Opportunistic Traffic Offloading Using Movement Predictions. In Proceedings of the 37th IEEE Conference on Local Computer Networks (LCN).

Bruce, Fette. 2007. Introducing Adaptive, Aware, and Cognitive Radios. In H. Arslan (Ed), Cognitive Radio, Software Defined Radio, and Adaptive Wireless Systems. Springer: The Netherlands. Biglieri Ezio, Robert Calderbank, Anthony Constantinides, Andrea Goldsmith, Arogyaswami Paulraj, H. Vincent Poor. 2010. MIMO Wireless Communications. Cambridge University Press. Castells, Manuel. 2009. Communication Power. New York: Oxford University Press.

Castells, Manuel. 1998. The Information Age: Economy, Society and Culture, Vol. III: End of Millennium. Malden MA. Oxford UK: Blackwell Publishers.

Chiani, Marco, Moe Z. Win, and Alberto Zanella. 2003. "On the capacity of spatially correlated MIMO Rayleigh-fading channels." Information Theory, IEEE Transactions 49.10: 2363-2371 Davies, Mike E., and Christopher J. James. 2007. "Source separation using single channel ICA." Signal Processing 87, no. 8: 1819-1832.

Haykin, Simon. 2005. “Cognitive radio: brain-empowered wireless communications.” Selected Areas in Communications, IEEE 23, no. 2: 201-220.

Jain, Mayank, Jung Il Choi, Taemin Kim, Dinesh Bharadia, Siddharth Seth, Kannan Srinivasan, Philip Levis, Sachin Katti, and Prasun Sinha. 2011. "Practical, real-time, full duplex wireless." In Proceedings of the 17th annual International Conference on Mobile Computing and Networking, pp. 301-312.

Jeffrey, G. Andrews. 2005. "Interference cancellation for cellular systems: a contemporary overview.” Wireless Communications, IEEE 12, no. 2: 19-29.

Karl D. Stephan, Katina Michael, M. G. Michael, Laura Jacob, and Emily P. Anesta. 2012. "Social Implications of Technology: The Past, the Present, and the Future" Proceedings of the IEEE, Vol. 100.

Lee, Kyunghan, et al. 2010. "Mobile data offloading: how much can WiFi deliver?." Proceedings of the 6th International COnference. ACM.

López-Pérez, David, Alvaro Valcarce, Guillaume De La Roche, and Jie Zhang. 2009. "OFDMA femtocells: a roadmap on interference avoidance." Communications Magazine, IEEE 47, no. 9: 41-48. Lozano, A. and Jindal N. 2010. "Transmit diversity vs. spatial multiplexing in modern MIMO systems”. IEEE Trans. Wireless Commun. 9 (1): 186-197.

Mitola, Joseph, and Gerald Q. Maguire Jr. 1999. "Cognitive radio: making software radios more personal.” Personal Communications, IEEE 6, no. 4: 13-18.

Neel, James O’Daniell. 2006. “Analysis and design of cognitive radio networks and distributed radio resource management algorithms." PhD diss., Virginia Polytechnic Institute and State University, 2006.

Russell, Mark, and Gordon L. Stuber. 1995. "Interchannel interference analysis of OFDM in a mobile environment.” In Vehicular Technology Conference, IEEE 45th, vol. 2:820-824.

Shin, Soo Young, Hong Seong Park, and Wook Hyun Kwon. 2007. "Mutual interference analysis of IEEE 802.15. 4 and IEEE 802.11 b.” Computer Networks 51, no. 12: 3338-3353.

Tarhini, Chadi, and Tijani Chahed. 2007. "On capacity of OFDMA-based IEEE802. 16 WiMAX including Adaptive Modulation and Coding (AMC) and inter-cell interference." In Local \& Metropolitan Area Networks. LANMAN 15th IEEE Workshop. pp. 139-144.

Torbjörn, Ekman. 2002. "Prediction of mobile radio channels: modeling and design." 


\section{H. Koçak, et. al}

Turkle, Sherry. 2011. Alone Together: Why We Expect More from Technology and Less from Each Other. New York: Basic Books.

www.usf.edu

www.aku.edu.tr

www.lus.edu.ba

Yucek, Tevfik, and Hüseyin Arslan. 2009. "A survey of spectrum sensing algorithms for cognitive radio applications." Communications Surveys \& Tutorials, IEEE 11, no. 1: 116-130.

Zhao, Qing, and Brian M. Sadler. 2007. "A survey of dynamic spectrum access." Signal Processing Magazine, IEEE 24, no. 3: 79-89.

Zhang, Honggang, Xiaofei Zhou, Kamya Yekeh Yazdandoost, and Imrich Chlamtac. 2006. "Multiple signal waveforms adaptation in cognitive ultra-wideband radio evolution." Selected Areas in Communications, IEEE 24, no. 4: 878-884. 